> Les récepteurs couplés aux protéines G (RCPG) constituent une famille de cibles thérapeutiques incontournables associées à un large spectre de fonctions. Les molécules actuellement sur le marché du type agoniste ou antagoniste agissent via le site actif du récepteur ou site orthostérique. Plus récemment, des composés se liant à des sites distincts, dits sites allostériques, ont été décrits dans différentes classes de RCPG. Ces composés, nommés modulateurs allostériques, sont généralement dotés d'une meilleure sélectivité entre les récepteurs d'une même famille et sont capables de moduler spécifiquement la signalisation endogène du récepteur, ce qui les distingue des composés orthostériques conventionnels. L'objectif de cette revue est de souligner le potentiel thérapeutique de cette nouvelle classe de composés, en décrivant les propriétés pharmacologiques associées aux modulateurs allostériques, leur stratégie d'identification et les défis liés à leur développement. <

Né dans les années 1960 , le concept d'interaction allostérique décrit des interactions protéiques associant des sites d'interaction différents [1]. Ce concept fondateur, qui englobe une multitude de mécanismes moléculaires du vivant, trouve en particulier des applications dans le domaine pharmacologique avec le développement de principes actifs interagissant avec différentes familles de récepteurs membranaires [2]. Environ $30 \%$ des médicaments sur le marché agissent sur des récepteurs couplés aux protéines $G$ (RCPG), cette famille de gènes étant donc la plus ciblée [3]. De plus, cette famille constitue un important réservoir de cibles potentielles [4] dont une part significative n'a pas encore de ligand endogène identifié [5]. Les différentes catégories de principes actifs développés à ce jour contre des RCPG agissent essentiellement au niveau du site de liaison au récepteur ou site orthostérique (Tableau I) [6]. Dans une

\section{Ciblage \\ thérapeutique des \\ récepteurs couplés \\ aux protéines G}

La voie allostérique

Julien A. Sebag ${ }^{1}$, Jacques Pantel ${ }^{1,2}$

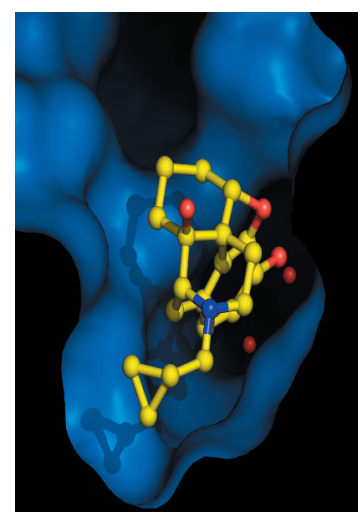

${ }^{1}$ Department of molecular physiology and biophysics, Vanderbilt School of Medicine, Nashville, TN, États-Unis ;

${ }^{2}$ Centre de psychiatrie et neurosciences, Inserm U894, 2 ter, rue d’Alésia, 75014 Paris, France. jacques.pantel@inserm.fr

autre famille de récepteurs, le concept de principe actif se liant à un site distinct du site orthostérique, dit site allostérique, a pourtant été largement popularisé en thérapeutique avec la classe des benzodiazépines, modulateurs allostériques du récepteur à l'acide $\gamma$-aminobutyrique de type $A\left(G A B A_{A}\right)[7]$. Dernièrement, des principes actifs allostériques ont été découverts pour différentes classes de RCPG [8], ce qui suggère qu'une propriété intrinsèque de cette famille de récepteurs est de pouvoir être ciblée par des modulateurs allostériques spécifiques. Dans cette revue, nous nous intéresserons tout particulièrement à cette nouvelle classe de principes actifs pour les RCPG et aux perspectives thérapeutiques qui en découlent (Tableau I).

\section{Mode d'action des modulateurs allostériques des RCPG}

La liaison du composé allostérique au récepteur, à distance du site orthostérique, va induire des modifications conformationnelles susceptibles de moduler la réponse fonctionnelle du ligand orthostérique endogène $[9,10]$. Ce mode d'action permissif par rapport à la molécule endogène se distingue du mode d'action compétitif des principes actifs orthostériques (Figure 1). L'attractivité thérapeutique des composés allostériques repose donc sur un effet modulateur qui s'exerce uniquement en présence de la molécule endogène : il s'agit de la modulation ciblée d'une signalisation endogène de RCPG. Ce mécanisme devrait en théorie préserver les rythmes physiologiques de signalisation du récepteur au cours du temps et selon le territoire d'activation. Ceci le distingue du principe actif orthostérique qui interfère de façon indifférenciée avec le récepteur, 
Site orthostérique

Ligands endogènes

(neuromédiateurs,

peptides, hormones, lipides, ions)
Agonistes (la plupart des cas)

Antagonistes et agonistes inverses

(agouti-related-protein $[\mathrm{AgRP}] /$ récepteurs mélanocortine [28])
Principes actifs

(agents pharmacologiques)
Agonistes

Agonistes inverses

Antagonistes

\section{Site allostérique}

5 - $\mathrm{HT}$ moduline/récepteur sérotonine $5-\mathrm{HT}_{1 \mathrm{~B}}$ [29]

Autres exemples dans [9]

Modulateurs allostériques

Tableau I. Catégories de molécules ciblant les RCPG en fonction de leur site d'interaction sur le récepteur.
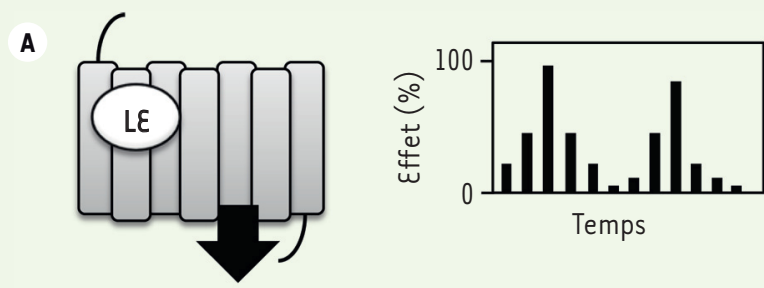

Temps

B
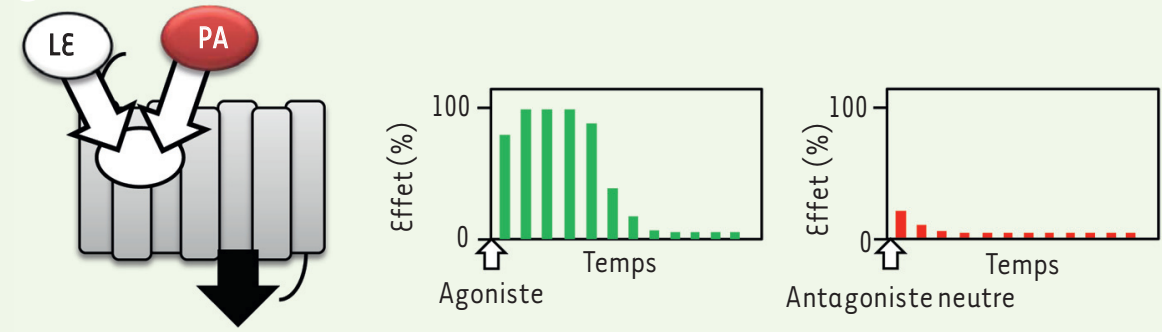

C

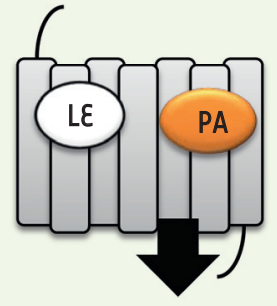

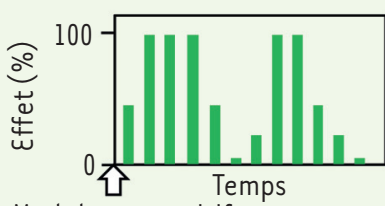

Modulateur positif
Antagoniste neutre
Modulateurnégatif

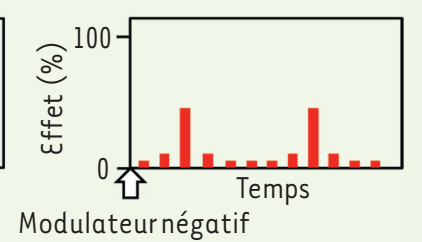

Figure 1. Mode d'action d'agents pharmacologiques orthostériques et allostériques et effets attendus sur la signalisation endogène d'un RCPG. Représentation au cours du temps du profil d'activation du RCPG en réponse à deux stimulations successives du ligand endogène $(L E)$ dans des conditions physiologiques $(A)$ ou en présence de doses efficaces de principes actifs (PA) orthostériques (B) ou allostériques (C). Les principes actifs orthostériques (agonistes ou antagonistes) illustrent un mode d'action compétitif qui interfère directement avec la signalisation endogène. La diminution du signal sous l'effet soutenu de l'agoniste représente un possible mécanisme de désensibilisation de la signalisation. Les principes actifs allostériques préservent le profil d'activation physiologique tout en le modulant positivement (tracé vert) ou négativement (tracé rouge).

quel que soit son degré de stimulation par la signalisation endogène (Figure 1).

Sur le plan de l'évolution, le site orthostérique d'un RCPG est naturellement soumis à une pression sélective favorisant sa conservation, parallèlement à celle du ligand endogène synonyme de fonction biologique. $\varepsilon$ n conséquence, dans le cas des RCPG comportant plusieurs sous-types de récepteurs reconnaissant le même ligand, le site orthostérique sera relativement conservé d'où la difficulté de découvrir des composés orthostériques très sélectifs (cas des récepteurs muscariniques de l'acétylcholine ou métabotropes du glutamate). En revanche, dans le cas des modulateurs allostériques, le site et/ou les mécanismes de coopérativité présidant à l'effet du modulateur ne sont pas a priori conservés entre les sous-types du récepteur, d'où la possibilité pour ces composés d'atteindre un haut degré de sélectivité (Figure 2), ce qui leur confère un avantage décisif sur les composés orthostériques.

\section{Propriétés des modulateurs allostériques}

Des modèles théoriques ont été proposés pour décrire l'interaction allostérique au niveau d'un RCPG selon un modèle de complexe ternaire allostérique se basant sur les constantes de dissociation du ligand endogène et du modulateur allostérique, ainsi que sur différentes constantes de coopérativité mesurant l'impact du modulateur allostérique sur l'affinité et 
A

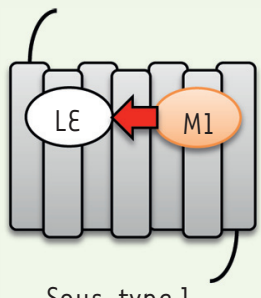

Sous-type 1

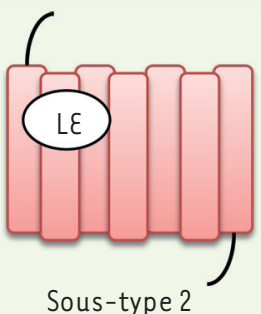

Sous-type 2

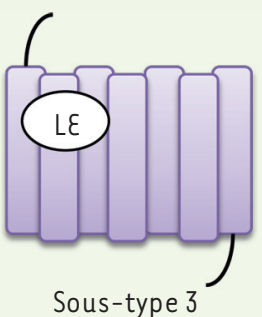

Sous-type 3

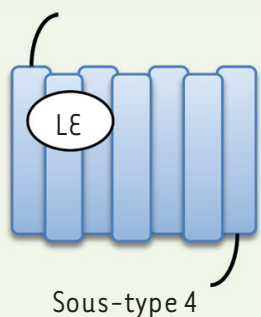

Sous-type 4

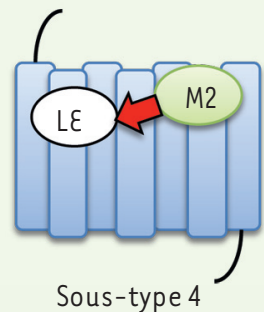

Sous-type 4

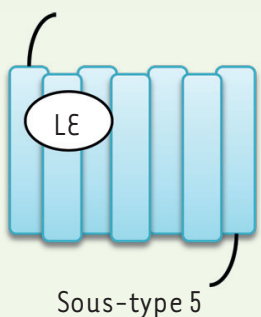

Sous-type 5

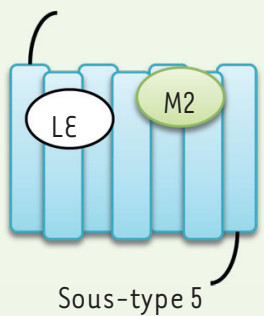

B

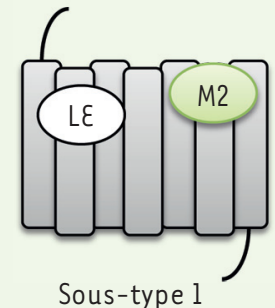

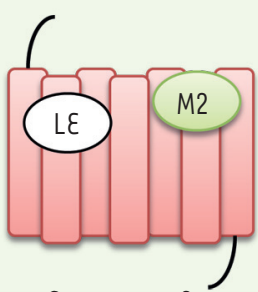

Sous-type 2

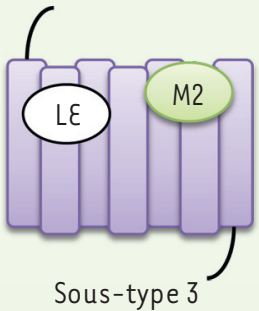

Figure 2. Sélectivité des modulateurs allostériques. Dans cette famille de RCPG répondant à un même ligand endogène (LE), le site orthostérique est conservé entre les différents sous-types (ovale blanc). Deux exemples de modulateurs allostériques (Ml et M2) décrivant des mécanismes de sélectivité différents sont représentés. L'effet du modulateur allostérique sur la signalisation du ligand endogène est représenté par une flèche rouge. $A$. Seul le sous-type 1 du récepteur est associé à la liaison et à l'effet allostérique du composé Ml, le site de liaison allostérique reconnu par Ml (ovale orange) n'est pas conservé dans les autres sous-types. La sélectivité de ce composé repose donc en grande partie sur l'affinité du composé pour le récepteur [9]. B. Le composé M2 se lie indifféremment aux différents sous-types du récepteur. Alors que le site allostérique reconnu par M2 (ovale vert) est conservé, seul le sous-type 4 est associé avec l'effet allostérique du modulateur. Dans ce cas, la sélectivité du modulateur repose sur un mécanisme de coopérativité, mécanisme décrit pour la première fois dans la famille des récepteurs muscariniques [30].

l'efficacité du ligand endogène (Figure 3A) [11]. En conséquence, le complexe formé du modulateur allostérique et du RCPG peut être considéré comme un nouveau récepteur pour son ligand endogène. En règle générale, selon la résultante de l'effet sur la signalisation du ligand endogène, les composés allostériques sont classés en modulateurs allostériques positifs ou négatifs [8-10]. Ces effets sont parfois associés à un effet propre du type agoniste ou agoniste inverse, selon l'aptitude du composé à stabiliser la forme active ou inactive du récepteur [12].

Le mécanisme d'action des composés allostériques leur donne des propriétés modulatrices particulières sur la signalisation endogène [8-10]. Premièrement, l'effet maximal du modulateur allostérique peut être saturable. Dans ce cas, quel que soit l'excès en modulateur, l'effet reste plafonné. Par opposition, l'effet propre du principe actif orthostérique, directement corrélé à sa concentration, lui confère une fenêtre thérapeutique plus étroite et un risque de désensibilisation du récepteur plus élevé. Deuxièmement, les propriétés modulatrices d'un composé allostérique sont spécifiques du ligand orthostérique; elles découlent de la coopérativité entre la paire des deux ligands dans un test donné. Ceci est tout particulièrement relevant dans des systèmes ou le RCPG est la cible de plusieurs ligands différents et ou le modulateur allostérique pourrait exercer des effets sélectifs différentiels. Troisièmement, les composés allostériques peuvent permettre de modifier les propriétés de puissance et d'efficacité du ligand orthostérique endogène de façon dissociée : certains composés auront un effet sur la puissance, d'autres sur l'efficacité, et d'autres enfin sur les deux paramètres conjointement (Figure 3B). Enfin, le changement de conformation du complexe RCPG-modulateur allostérique peut favoriser une voie de signalisation au détriment d'une autre. Ce phénomène, nommé sélectivité fonctionnelle ou signalisation biaisée, est susceptible d'avoir des applications dans le développement de médicaments pour, par exemple, favoriser une voie de signalisation associée à un effet thérapeutique tout en bloquant une voie de signalisation indésirable associée à des effets secondaires.

\section{Identification de modulateurs allostériques d'un RCPG par criblage}

L'histoire des modulateurs allostériques concernant les RCPG est étroitement associée aux progrès technologiques du criblage cellulaire à haut débit au cours de cette dernière dizaine d'années. Ces coûteuses approches de criblage visent à identifier parmi les 
A

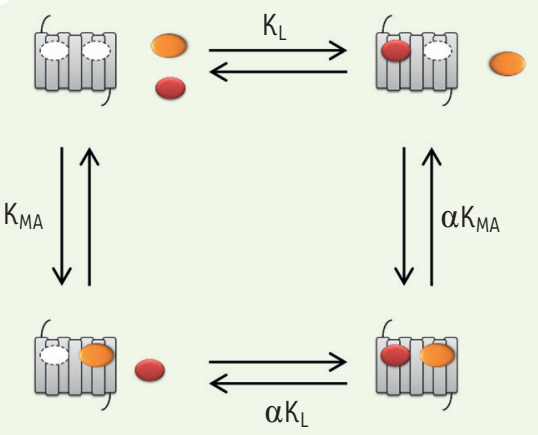

B

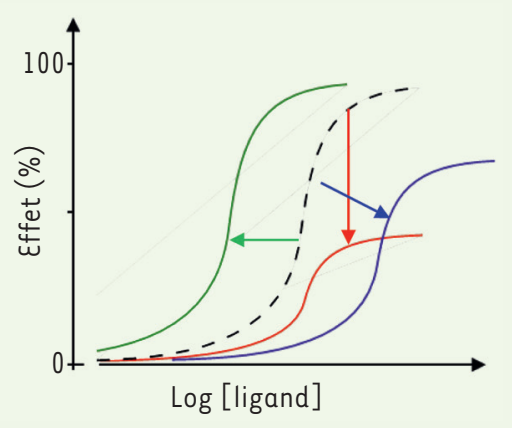

Figure 3. Interaction allostérique : modélisation et conséquences fonctionnelles. A. Complexe ternaire servant de base à la modélisation de l'interaction d'un RCPG (en gris) avec un ligand endogène orthostérique (ovale rouge) et un modulateur allostérique (ovale orange). Les équilibres entre les différents états sont décrits par les constantes de dissociation du ligand endogène au récepteur et du modulateur au récepteur,

notées $K_{L}$ et $K_{M A}$ respectivement. Des constantes de coopérativité et d'efficacité ont été développées pour prendre en compte l'impact du modulateur allostérique sur l'affinité $(\alpha)$ et/ou l'efficacité du ligand. B. Modification de la réponse pharmacologique du ligand endogène d'un RCPG par un modulateur allostérique dans un test fonctionnel donné. La courbe de réponse du ligand en fonction de sa concentration en l'absence de modulateur allostérique est représentée en pointillés. Les flèches de couleur illustrent les modifications de cette courbe en présence de concentrations efficaces de différents exemples de modulateurs allostériques : un modulateur allostérique positif augmentant la puissance (vert), deux modulateurs allostériques négatifs diminuant l'efficacité (rouge) ou diminuant conjointement l'efficacité et la puissance (bleu).

composés issus d'une large chimiothèque, lesquels sont potentiellement actifs sur le RCPG ciblé au moyen d'un test spécifique. Chronologiquement, les tests basés sur le déplacement d'un ligand radiomarqué au site orthostérique du RCPG ont naturellement favorisé la détection d'agonistes et d'antagonistes orthostériques. Plus récemment, l'avènement de tests fonctionnels qui évaluent la réponse biologique associée au RCPG a élargi le spectre des composés découverts aux composés allostériques. Idéalement, ces tests fonctionnels devraient mimer des réponses cellulaires les plus physiologiques possibles tout en respectant les contraintes inhérentes au ciblage à haut débit. Malgré des progrès significatifs dans le domaine de l'instrumentation, ces deux types de critères restent difficiles à concilier. En pratique, les tests de criblage se basent essentiellement sur des lignées cellulaires, ce qui complique la transposition des résultats à des conditions plus physiologiques.

Le criblage à haut débit comporte schématiquement trois aspects : les composés chimiques à tester, le test de criblage et enfin la méthodologie propre au haut débit. Premièrement, les composés chimiques à tester sont issus de chimiothèques de petites molécules représentant une large diversité chimique que possèdent les laboratoires pharmaceutiques, mais aussi désormais certains laboratoires académiques [13]. Deuxièmement, le test de criblage de choix est un test cellulaire permettant d'enregistrer la réponse fonctionnelle du RCPG d'intérêt. Au plan pratique, les tests enregistrant une réponse fonctionnelle en temps réel sont préférés puisqu'ils présentent l'avantage, selon le protocole choisi, de pouvoir sélectionner modulateurs agonistes et/ou antagonistes en un test unique (Figure 4A), et donc d'optimiser les résultats d'un criblage. Parmi ceux-ci, les mesures de flux calcique occupent une place de choix puisqu'elles peuvent permettre de détecter la réponse fonctionnelle associée à l'activation de RCPG couplés aux protéines $G_{\alpha q / 11}$ ou à d'autres sous- types par des artifices moléculaires [14]. Récemment, un rapporteur luminescent sensible à l'AMP cyclique $(A M P c)$ a été validé dans un test fonctionnel en temps réel au format à haut débit pour identifier des modulateurs allostériques d'un récepteur couplé aux protéines $G_{\alpha s}$, le récepteur mélanocortine 4 (MC4R) [15]. Troisièmement, le test fonctionnel du RCPG doit se conformer aux contraintes méthodologiques du haut débit, qui mettent en œuvre un appareillage de haute technicité pour faire face au nombre de composés à cribler qui évolue entre des centaines de milliers à des millions. Les notions de qualité, de reproductibilité et de coût du test entrent en ligne de compte dans le choix final du test et du protocole de criblage [16]. En particulier, un contrôle de qualité approprié au criblage à haut débit a été développé spécifiquement pour valider la qualité du test, puis les séries de données produites au cours de la campagne de criblage (Figure 4B) [17].

Chronologiquement, le criblage primaire constitue l'étape clé dont dépend le succès de l'approche puisqu'il doit permettre, en un seul test, d'identifier des composés actifs au sens large, tout en restant suffisamment sélectif pour éviter un nombre trop élevé de composés (1-5\% du nombre total de composés). À l'issue du criblage primaire, le nombre de composés d'intérêt a dramatiquement diminué permettant cette fois de réaliser une exploration fonctionnelle plus fine pour éliminer les faux positifs et les composés non spécifiques du RCPG. Un travail de classement des composés sur la base de leur 
A

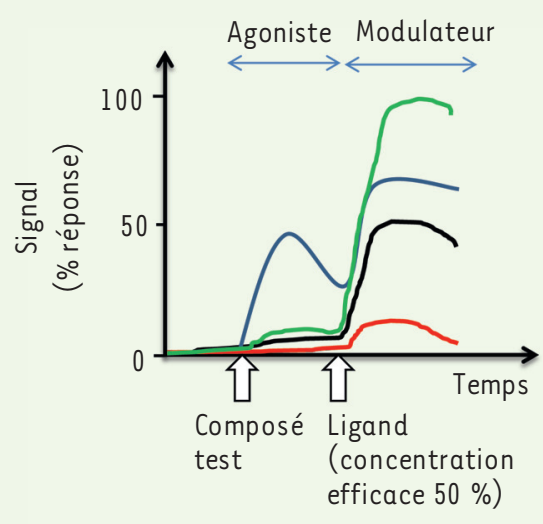

B

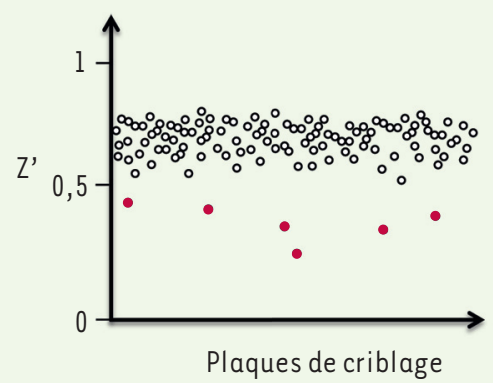

Optimisation

C

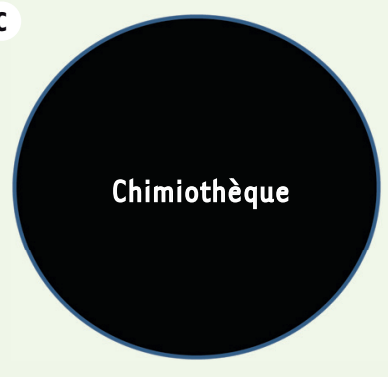

$\mathrm{n}=10^{5}-10^{6}$
Criblage à haut débit

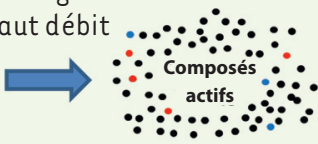

$\mathrm{n}=10^{2}-10^{3}$
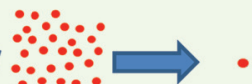
$\because \because \because \because$

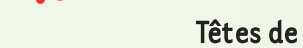

séries

$\mathrm{n}<3$
Figure 4. Identification de modulateurs allostériques d'un RCPG par une approche de criblage à haut débit. A. Test de criblage allostérique basé sur la réponse en temps réel d'un RCPG. Pour favoriser la découverte de composés allostériques, le composé test est mis en présence d'un ligand orthostérique, de préférence le ligand endogène. Dans chaque puits réactionnel, le lecteur de microplaques enregistre en temps réel le signal émis au niveau basal, le signal après l'addition du composé testé (en général à $10 \mu \mathrm{M})$, puis après l'addition du ligand orthostérique à une concentration induisant $50 \%$

de l'effet maximal $\left(\varepsilon C_{50}\right)$. Sont représentés les patrons de réponse d'une sélection de composés tests compatibles avec un agoniste (bleu), un modulateur positif (vert), un modulateur négatif (rouge) ou un composé inactif (noir). B. Contrôles de qualité du criblage à haut débit. Du fait de l'extrême complexité d'un criblage HTS (high-throughput screening) (dimensions biologiques, physiques, robotiques), un paramètre statistique ( $\left.Z^{\prime}\right)$ a été développé pour mesurer la qualité du test à un moment donné [17]. Ce paramètre, compris entre 0 et 1 , décrit la variance entre les signaux bas et les signaux élevés d'un test de criblage, une valeur de 0,5 étant considérée comme compatible avec le criblage à haut débit. Ce paramètre est employé au cours de l'optimisation du test de criblage au format haut débit et, dans le cas présenté ici, comme contrôle de qualité. Les plaques de composés ne satisfaisant pas aux critères de qualité sont à repasser (points rouges). C. Organigramme des étapes précoces de la découverte de nouvelles entités chimiques actives par criblage à haut débit. Le criblage à haut débit permet de tester l'activité d'un grand nombre de composés dans le test unique de criblage, pour ne retenir que la fraction des composés dits actifs. Une fois l'activité et la spécificité de ces composés vérifiées (expériences de réplication et de contre-criblage), un classement des composés d'intérêt par efficacité et par structure chimique est dressé dans le but d'identifier en priorité des composés actifs partageant une structure chimique voisine pour n'en retenir qu'un nombre très restreint (points de couleur). Ces ébauches de relations structure-fonction seront alors approfondies par la synthèse d'une collection de dérivés par famille dont l'activité sera évaluée dans plusieurs tests. Ce travail conduira à la sélection d'une molécule originale dite «tête de série » active dans plusieurs tests fonctionnels secondaires, biologiques et ou phénotypiques, et dotée de propriétés physicochimiques acceptables pour les étapes ultérieures de développement.

structure chimique et de leurs propriétés in vitro visera à identifier des ébauches de relations structure-fonction (Figure 4C). Celui-ci sera suivi par un travail de chimie médicinale qui va aboutir à des composés optimisés (amélioration des paramètres de puissance, de sélectivité et de toxicité). Au final, les relations structure-fonction obtenues par cette approche, éventuellement associées à de la modélisation, permettent d'orienter la recherche vers des structures chimiques candidates.

\section{Défis liés au développement des modulateurs allostériques des RCPG}

Bien que les modulateurs allostériques présentent des propriétés prometteuses en thérapeutique, un certain nombre de questions spécifiques se posent au cours des différentes étapes qui mènent de leur découverte à leur traduction en principes actifs. 
Premièrement, les mécanismes de coopérativité associés aux modulateurs allostériques les rendent potentiellement plus vulnérables à d'autres interactions moléculaires mettant en jeu les RCPG, dont de plus en plus d'exemples illustrent la signification physiologique : dimérisation ou oligomérisation des $\operatorname{RCPG}[18,31]$ $(\rightarrow)$, protéines accessoires [19], ou signalisation biaisée $[20,32](\rightarrow)$. Or, cet interactome n'est pas pris en compte à ce jour dans les approches de criblages à haut débit menées sur les systèmes recombinants [21]. En effet, au vu des données sur

$\rightarrow$ Voir l'article de R. Jockers et al., page 864 de ce numéro

$\rightarrow$ Voir l'article de C. Galès, page 883 de ce numéro

l'ensemble des médicaments mis sur le marché ces dix dernières années, l'efficacité de l'approche de criblage ciblée pour découvrir de nouvelles classes de médicaments est distancée par des approches plus physiologiques (phénotypiques et biologiques). En revanche, cette approche est de loin la plus efficace pour découvrir de nouveaux médicaments dans une classe préexistante grâce à ses fondements moléculaires [22]. En conséquence, et encore plus particulièrement pour les modulateurs allostériques, la sélection de composés efficaces in vivo requiert leur validation, le plus tôt possible après le criblage primaire, dans des tests secondaires qui se rapprochent le plus du plan physiologique.

Deuxièmement, la classification fonctionnelle des modulateurs allostériques peut se révéler plus complexe que celle des composés orthostériques du fait de l'importance toute particulière du contexte dans la nature de l'effet (ligands endogènes, voie de signalisation, système cellulaire) [23].

Troisièmement, l'effet fonctionnel d'un composé allostérique identifié sur un RCPG humain ne sera pas nécessairement conservé sur les orthologues d'autres espèces, ce qui peut éventuellement constituer un obstacle pour réaliser des tests d'efficacité dans le modèle animal correspondant [8].

Quatrièmement, l'optimisation de ces composés par les approches de chimie médicinale est rendue plus délicate par l'existence de switchs moléculaires pouvant conduire indifféremment à des composés silencieux ou à des réversions de l'effet (modulateurs positifs en modulateurs négatifs, ou inversement) [24].

\section{Perspectives thérapeutiques}

À ce jour, validant le concept de l'efficacité de modulateurs allostériques de RCPG au plan thérapeutique, deux composés ont atteint la mise sur le marché. Le premier composé, le cinacalcet, principe actif spécifique du calcium-sensing receptor (CaSR), est le premier traitement médicamenteux proposé pour l'hyperparathyrö̈die [25]. Ce composé est un modulateur allostérique positif qui agit par une augmentation de la puissance du ligand endogène, le calcium extracellulaire, pour le CaSR. La découverte de ce site allostérique propose en outre une alternative au ciblage de ce RCPG dont le site orthostérique reconnaissant un ion inorganique est difficilement accessible par nature. Le second composé, le maraviroc, dirigé contre le récepteur de chimiokine-5 (CCR5), est un traitement anti-VIH (virus de l'immunodéficience humaine) [26]. La liaison de ce modulateur allostérique négatif au CCR5 conduit à un récepteur d'affinité réduite

pour la protéine d'enveloppe du VIH, ce qui aboutit au blocage de l'entrée du VIH dépendante du CCR5 dans les cellules. La découverte du maraviroc illustre le succès d'une approche de criblage à haut débit ciblée sur un RCPG et couplée à une vaste campagne d'optimisation. Actuellement, un nombre croissant de composés allostériques en cours de développement ont démontré leur efficacité dans des tests précliniques compatibles notamment avec le traitement de certaines maladies du système nerveux central (anxiété, schizophrénie, maladies d'Alzheimer et de Parkinson) [8, 27]. Le succès de ces travaux de recherche s'illustre d'ores et déjà dans l'éventail des molécules ayant atteint un degré inégalé de sélectivité dans les familles de RCPG dont la trop forte homologie avait jusqu'alors tenu en échec la perspective d'obtenir des molécules sélectives (cas des récepteurs muscariniques et métabotropiques) [8]. Ceci offre, de fait, des perspectives pour d'autres familles de RCPG. Cependant, il est encore trop tôt pour apprécier l'importance réelle du transfert thérapeutique de ces nouvelles classes de principes actifs.

\section{Conclusion}

La découverte récente de modulateurs allostériques dans différentes classes de RCPG propose des principes actifs dotés de propriétés originales susceptibles de renouveler l'offre des composés orthostériques, mais aussi de cibler des sous-types de récepteurs jusqu'à présent inaccessibles. Ces composés devraient permettre de proposer des principes actifs plus sélectifs, de diminuer certains effets indésirables associés aux composés orthostériques et d'élargir le champ thérapeutique à certaines maladies complexes touchant notamment le système nerveux central. Les données actuelles laissent entrevoir le transfert en thérapeutique d'un nombre croissant de modulateurs allostériques de RCPG dans un futur proche. $\diamond$

\section{SUMMARY}

G-protein-coupled receptors targeting: the allosteric approach

G-protein-coupled receptors (GPCR) are a major family of drug targets. Essentially all drugs targeting these receptors on the market compete with the endogenous ligand (agonists or antagonists) for binding the receptor. Recently, non-competitive compounds binding to distinct sites from the cognate ligand were documented in various classes of these receptors. These compounds, called allosteric modulators, generally endowed of a better selectivity are able to modulate specifically the endogenous signaling of the receptor. To better unders- 
tand the promising potential of this class of GPCRs targeting compounds, this review highlights the properties of allosteric modulators, the strategies used to identify them and the challenges associated with the development of these compounds. $\diamond$

\section{LIENS D'INTÉRÊT}

Les auteurs déclarent n'avoir aucun lien d'intérêt concernant les données publiées dans cet article.

\section{RÉFÉRENCES}

1. Monod J, Wyman J, Changeux JP. On the nature of allosteric transitions: a plausible model.J Mol Biol 1965; $12: 88-118$.

2. Changeux JP. Allosteric receptors: from electric organ to cognition. Annu Rev Pharmacol Toxicol $2010 ; 50: 1-38$

3. Overington JP, Al-Lazikani B, Hopkins AL. How many drug targets are there? Nat Rev Drug Discov $2006 ; 5: 993-6$

4. Lagerstrom MC, Schioth HB. Structural diversity of $G$ protein-coupled receptors and significance for drug discovery. Nat Rev Drug Discov 2008 ; 7 : 339-57.

5. Chung S, Funakoshi T, Civelli O. Orphan GPCR research. BrJ Pharmacol 2008 ; 153 : S339-46.

6. Hopkins AL, Groom CR. The druggable genome. Nat Rev Drug Discov $2002 ; 1: 727-30$.

7. Sigel $\varepsilon$. Mapping of the benzodiazepine recognition site on GABA(A) receptors. Curr Top Med Chem $2002 ; 2: 833-9$

8. Conn PJ, Christopoulos A, Lindsley CW. Allosteric modulators of GPCRs: a novel approach for the treatment of CNS disorders. Nat Rev Drug Discov 2009; $8: 41-54$.

9. May LT, Leach K, Sexton PM, Christopoulos A. Allosteric modulation of G protein-coupled receptors. Annu Rev Pharmacol Toxicol 2007 ; $47: 1-51$.

10. Kenakin T, Miller LJ. Seven transmembrane receptors as shapeshifting proteins: the impact of allosteric modulation and functional selectivity on new drug discovery. Pharmacol Rev 2010 ; $62: 265-304$

11. Hall DA. Modeling the functional effects of allosteric modulators at pharmacological receptors: an extension of the two-state model of receptor activation. Mol Pharmacol $2000 ; 58: 1412-23$.

12. Smit MJ, Vischer HF, Bakker RA, et al. Pharmacogenomic and structural analysis of constitutive $G$ protein-coupled receptor activity. Annu Rev Pharmacol Toxicol 2007 ; 47 : 53-87.

13. Macarron R, Banks MN, Bojanic D, et al. Impact of high-throughput screening in biomedical research. Nat Rev Drug Discov 2011 ; 10 : 188-95.

14. Emkey R, RankI NB. Screening $G$ protein-coupled receptors: measurement of intracellular calcium using the fluorometric imaging plate reader. Methods Mol Biol $2009 ; 565: 145-58$.

15. Pantel J, Williams SY, Mi D, et al. Development of a high throughput screen for allosteric modulators of melanocortin-4 receptor signaling using a real time CAMP assay. Eur J Pharmacol $2011 ; 660: 139-47$.

16. Hughes JP, Rees S, Kalindjian SB, Philpott KL. Principles of early drug discovery. Br J Pharmacol $2011 ; 162: 1239-49$.

17. Zhang JH, Chung TD, Oldenburg KR. A simple statistical parameter for use in evaluation and validation of high throughput screening assays. J Biomol Screen 1999; 4:67-73.
18. Bouvier M. Oligomerization of G-protein-coupled transmitter receptors. Nat Rev Neurosci $2001 ; 2: 274-86$.

19. Bockaert J, Perroy J, Becamel C, et al. GPCR interacting proteins (GIP) in the nervous system: roles in physiology and pathologies. Annu Rev Pharmacol Toxicol $2010 ; 50: 89-109$.

20. Rajagopal S, Rajagopal K, Lefkowitz RJ. Teaching old receptors new tricks: biasing seven-transmembrane receptors. Nat Rev Drug Discov 2010 ; 9 : 373-86.

21. Milligan G, Smith NJ. Allosteric modulation of heterodimeric G-proteincoupled receptors. Trends Pharmacol Sci 2007 ; 28 : 615-20.

22. Swinney DC, Anthony J. How were new medicines discovered? Nat Rev Drug Discov $2011 ; 10: 507-19$.

23. Keov P, Sexton PM, Christopoulos A. Allosteric modulation of G proteincoupled receptors: a pharmacological perspective. Neuropharmacology $2011 ; 60: 24-35$.

24. Wood MR, Hopkins CR, Brogan JT, et al. Molecular switches on mGluR allosteric ligands that modulate modes of pharmacology. Biochemistry $2011 ; 50: 2403-10$.

25. Harrington $P E$, Fotsch C. Calcium sensing receptor activators: calcimimetics. Curr Med Chem 2007 ; 14 : 3027-34.

26. Dorr P, Westby M, Dobbs S, et al. Maraviroc (UK-427,857), a potent, orally bioavailable, and selective small-molecule inhibitor of chemokine receptor CCR5 with broad-spectrum anti-human immunodeficiency virus type 1 activity. Antimicrob Agents Chemother $2005 ; 49$ : 4721-32.

27. Urwyler S. Allosteric modulation of family C G-protein-coupled receptors: from molecular insights to therapeutic perspectives. Pharmacol Rev 2011 ; $63: 59-126$.

28. Ollmann MM, Wilson BD, Yang YK, et al. Antagonism of central melanocortin receptors in vitro and in vivo by agouti-related protein. Science $1997 ; 278$ $135-8$.

29. Massot 0, Rousselle JC, Fillion MP, et al. 5-hydroxytryptamine-moduline, a new endogenous cerebral peptide, controls the serotonergic activity via its specific interaction with 5-hydroxytryptaminelB/1D receptors. Mol Pharmacol $1996 ; 50: 752-62$

30. Lazareno S, Dolezal V, Popham A, Birdsall NJ. Thiochrome enhances acetylcholine affinity at muscarinic M4 receptors: receptor subtype selectivity via cooperativity rather than affinity. Mol Pharmacol $2004 ; 65$ : 257-66.

31. Jockers R, Gbahou F, Kamal M, Tadagaki K. Oligomérisation des protéines humaines et virales à 7 domaines transmembranaires : nouvelle stratégie virale pour manipuler la cellule hôte. Med Sci (Paris) 2012 ; 28 : 864-9.

32. Galès $C$. Plasticité des récepteurs couplés aux protéines $G$ et signalisation : un Rubik's cube. Med Sci (Paris) $2012 ; 28: 883-5$.

\section{TIRÉS À PART}

J. Pantel

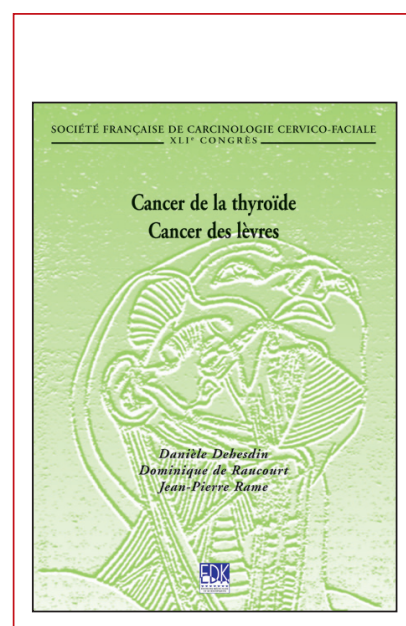

ISBN : 978-2-8425-4137-8 264 pages

\section{Bon de commande}

À retourner à EDK, 25, rue Daviel - 75013 Paris

Tél. : 0158101905 - Fax : 0143293262 - E-mail : edk@edk.fr

NOM :

Prénom :

Adresse :

Code postal :

Ville :

Pays :

Fonction :

Je souhaite recevoir l'ouvrage Cancer de la thyroïde - Cancers des lèvres : $35 €+3 €$ de port $=\mathbf{3 8} €$ TTC

en ............... exemplaire, soit un total de ...................................... €

$\square$ Par chèque, à l'ordre de $\mathbf{E} \mathbf{D} \mathbf{K}$

$\square$ Par carte bancaire : $\square$ Visa $\square$ Eurocard/Mastercard

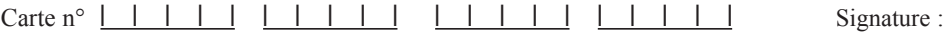

Date d'expiration: $\quad \underline{1} \mid \underline{1}$

$\mathrm{N}^{\circ}$ de contrôle au dos de la carte : $\quad \underline{\text { | }}$ | | | | 